\title{
Improved shear correction factors for deflection of simply supported very thick rectangular auxetic plates
}

T. C. Lim

\begin{abstract}
Background: The first-order shear deformation theory (FSDT) for plates requires a shear correction factor due to the assumption of constant shear strain and shear stress across the thickness; hence, the shear correction factor strongly influences the accuracy of the deflection solution; the third-order shear deformation theory (TSDT) does not require a correction factor because it facilitates the change in shear strain across the plate thickness.
\end{abstract}

Methods: This paper obtains an improved shear correction factor for simply supported very thick rectangular plates by matching the deflection of the Mindlin plate (FSDT) with that of the Reddy plate (TSDT).

Results: As a consequence, the use of the exact shear correction factor for the Mindlin plate gives solutions that are exactly the same as for the Reddy plate.

Conclusions: The customary adoption of 5/6 shear correction factor is a lower bound, and the exact shear correction factor is higher for the following: (a) very thick plates, (b) narrow or long plates, (c) high Poisson's ratio plate material, and (d) highly patterned loads, while the commonly used shear correction factor of 5/6 is still valid for the following: (i) marginally thick plates, (ii) square plates, (iii) negative Poisson's ratio materials, and (d) uniformly distributed loadings.

Keywords: Aspect ratio, Auxetic materials, Incompressible materials, Shear deformation, Thick plates

\section{Background}

It is well known that the shear correction factors of plates are simpler than those for beams (Dong et al., 2010; Puchegger et al., 2003; Hlavacek and Chleboun, 2000; Pai and Schultz, 1999; Popescu and Hodges, 2000; $\mathrm{Yu}$ and Hodges, 2004; Chan et al., 2011; Pai et al., 2000; Hutchinson, 1980; Hutchinson, 2001; Han et al., 1999); this is due to the cross-sectional geometry in beams being more varied than for plates. For plates, the commonly adopted shear correction factor is typically $5 / 6$; in some instances, Poisson's ratio is taken into account (e.g., Rössle, 1999; Lee et al., 2002). Exact shear correction factors for vibrating Mindlin plates have been proposed by Stephen (1997) and Hull $(2005,2006)$. In this paper, exact shear correction factors for simply supported very thick rectangular Mindlin plates are derived by comparing its deflection against that of Reddy plates. The Mindlin plate,

Correspondence: alan_tc_lim@yahoo.com

School of Science and Technology, SIM University, Singapore, Singapore which adopts the first-order shear deformation theory (FSDT), requires a correction factor due to its assumption of uniform shear across the plate thickness while the Reddy plate, which adopts the third-order shear deformation theory (TSDT), does not require any correction as it caters for the varying shear strain across the plate thickness. The rigor of the Reddy plate, therefore, forms the justification for its use as a benchmark for evaluating the accuracy of Mindlin plate deflection-this has been done for triangular plates (Lim, 2016a, b). Following a recent preliminary analysis (Lim, 2016c) to evaluate the ratio of maximum deflection of Reddy plate to that of Kirchhoff plate or the classical plate theory (CPT), the TSDT is now being employed for extracting the exact shear correction factor of rectangular plates in the FSDT.

\section{Methods}

\section{General consideration}

Figure 1 illustrates a simply supported thick rectangular plate of sides $a$ and $b$, measured along the $x$ and $y$ axes, 

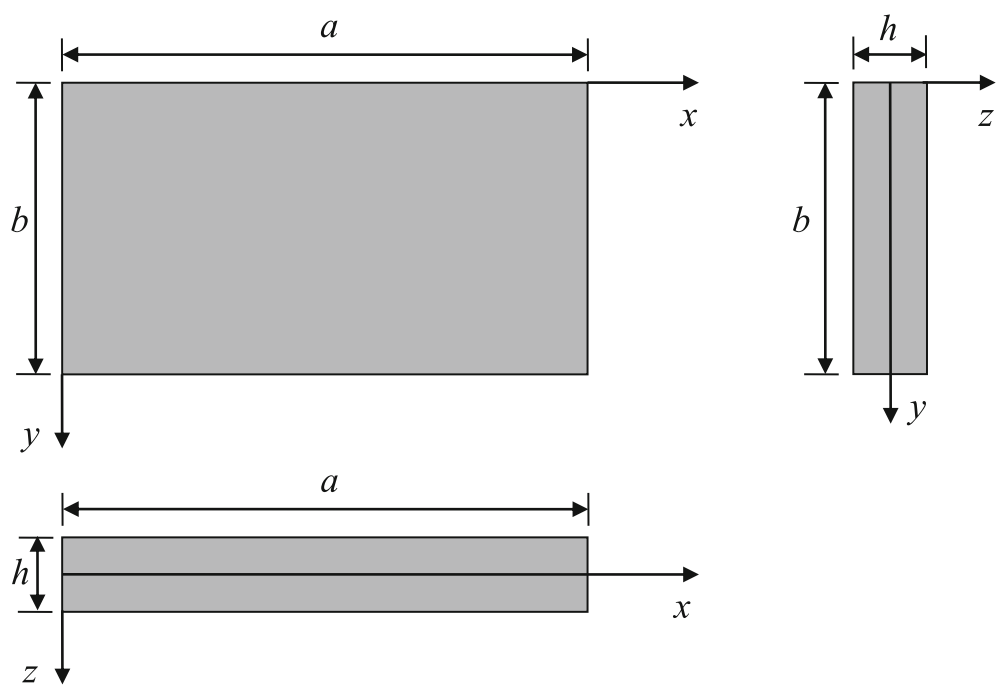

Fig. 1 Geometrical nomenclature adopted for analysis

respectively, while the thickness $h$ is measured along the $z$ axis. Recall that the Mindlin plate deflection $w^{M}$ is related to the Kirchhoff plate deflection $w^{\mathrm{K}}$ as

$$
w^{\mathrm{M}}=w^{\mathrm{K}}+\frac{M^{\mathrm{K}}}{\kappa G h}
$$

where the moment sum, or Marcus moment, is defined as

$$
M^{\mathrm{K}}=-D \nabla^{2} w^{\mathrm{K}}
$$

and $D$ is the plate flexural rigidity, while the Reddy plate deflection $w^{\mathrm{R}}$ is related to the Kirchhoff plate deflection as

$$
w^{\mathrm{R}}=w^{\mathrm{K}}+\frac{1}{G h}\left(\alpha C_{1} D \nabla^{2} w^{\mathrm{R}}+C_{4} M^{\mathrm{K}}\right)
$$

where $280 C_{1}=3 h^{2}=4 / \alpha, C_{4}=17 / 14$, and

$$
\lambda_{0}^{2}=\frac{G h}{\alpha C_{1} D}=\frac{70 G h}{D},
$$

with $G$ being the shear modulus of the plate.

With reference to Eqs.(1) and (2), it is useful to describe the plate flexural ridigity

$$
D=\frac{E h^{3}}{12\left(1-v^{2}\right)}
$$

and the shear modulus

$$
G=\frac{E}{2(1+v)},
$$

where $E$ is Young's modulus, as the ratio

$$
\frac{D}{G}=\frac{h^{3}}{6(1-v)}
$$

so that Eqs.(1) and (2) can be expressed as

$$
w^{\mathrm{M}}=w^{\mathrm{K}}-\frac{h^{2}}{6 \kappa(1-v)} \nabla^{2} w^{\mathrm{K}}
$$

and

$$
w^{\mathrm{R}}-\frac{h^{2}}{420(1-v)} \nabla^{2} w^{\mathrm{R}}=w^{\mathrm{K}}-\frac{17 h^{2}}{84(1-v)} \nabla^{2} w^{\mathrm{K}}
$$

respectively, to facilitate comparison. Equating the Mindlin and Reddy plate deflections gives the following general relationship under the same boundary condition:

$$
\frac{1}{\kappa}=\frac{17}{14}-\frac{1}{70} \frac{\Delta w^{\mathrm{R}}}{\Delta w^{\mathrm{K}}}
$$

where

$$
\Delta=\nabla^{2}=\frac{\partial^{2}}{\partial x^{2}}+\frac{\partial^{2}}{\partial y^{2}} .
$$

Perusal to Eq. (5a) suggests that a meaningful exact shear correction factor can be obtained if both the Reddy plate and Kirchhoff plate deflections are known. Neglecting the higher order term in Eq. (5a) gives a shear correction factor of $k=14 / 17$. The two constant shear correction factors of $5 / 6$ and $14 / 17$ have been discussed by Wang et al. (2000).

\section{Uniform load}

As the Kirchhoff plate deflection for a simply supported rectangular plate under uniform load $q=q_{0}$ is 


$$
w^{\mathrm{K}}=\frac{16 q_{0}}{\pi^{6} D} \sum_{m=1}^{\infty} \sum_{n=1}^{\infty} \frac{\sin \frac{m \pi x}{a} \sin \frac{n \pi y}{b}}{m n\left(\frac{m^{2}}{a^{2}}+\frac{n^{2}}{b^{2}}\right)^{2}}
$$

with $m, n=1,3,5, \ldots$, we adopt a similar profile deflection for the Reddy plate

$$
w^{\mathrm{R}}=A^{\mathrm{R}} \sum_{m=1}^{\infty} \sum_{n=1}^{\infty} \frac{\sin \frac{m \pi x}{a} \sin \frac{n \pi y}{b}}{m n\left(\frac{m^{2}}{a^{2}}+\frac{n^{2}}{b^{2}}\right)^{2}},
$$

where $A^{\mathrm{R}}$ is the amplitude term of the Reddy plate. Substituting the deflection profiles of Kirchhoff and Reddy plates into the relationships described by Eq. (4) leads to

$$
\begin{aligned}
w^{\mathrm{M}}=\frac{16 q_{0}}{\pi^{6} D}\left\{\sum_{m=1}^{\infty} \sum_{n=1}^{\infty} \frac{\sin \frac{m \pi x}{a} \sin \frac{n \pi y}{b}}{m\left(\frac{m^{2}}{a^{2}}+\frac{n^{2}}{b^{2}}\right)^{2}}\right. \\
\left.+\frac{\pi^{2} h^{2}}{6 \kappa(1-v)} \sum_{m=1}^{\infty} \sum_{n=1}^{\infty} \frac{\sin \frac{m \pi x}{a} \sin \frac{n \pi y}{b}}{m n\left(\frac{m^{2}}{a^{2}}+\frac{n^{2}}{b^{2}}\right)}\right\}
\end{aligned}
$$

and

$$
\begin{gathered}
\sum_{m=1}^{\infty} \sum_{n=1}^{\infty} \frac{\sin \frac{m \pi x}{a} \sin \frac{n \pi y}{b}}{m n\left(\frac{m^{2}}{a^{2}}+\frac{n^{2}}{b^{2}}\right)^{2}} \\
A^{\mathrm{R}=\frac{16 q_{0}}{\pi^{6} D}} \frac{17 \pi^{2} h^{2}}{84(1-v)} \sum_{m=1}^{\infty} \sum_{n=1}^{\infty} \frac{\sin \frac{m \pi x}{a} \sin \frac{n \pi y}{b}}{m n\left(\frac{m^{2}}{a^{2}}+\frac{n^{2}}{b^{2}}\right)} \\
\sum_{m=1}^{\infty} \sum_{n=1}^{\infty} \frac{\sin \frac{m \pi x}{a} \sin \frac{n \pi y}{b}}{m n\left(\frac{m^{2}}{a^{2}}+\frac{n^{2}}{b^{2}}\right)^{2}} \\
+\frac{\pi^{2} h^{2}}{420(1-v)} \sum_{m=1}^{\infty} \sum_{n=1}^{\infty} \frac{\sin \frac{m \pi x}{a} \sin \frac{n \pi y}{b}}{m n\left(\frac{m^{2}}{a^{2}}+\frac{n^{2}}{b^{2}}\right)}
\end{gathered}
$$

Introducing the function

$$
f(a, b, x, y)=\frac{\sum_{m=1}^{\infty} \sum_{n=1}^{\infty} \frac{\sin \frac{m \pi x}{a} \sin \frac{n \pi y}{b}}{m n\left(\frac{b}{a} m^{2}+\frac{a}{b} n^{2}\right)}}{\sum_{m=1}^{\infty} \sum_{n=1}^{\infty} \frac{\sin \frac{m \pi x}{a} \sin \frac{n \pi y}{b}}{m n\left(\frac{b}{a} m^{2}+\frac{a}{b} n^{2}\right)^{2}}}
$$

allows Eq. (9) to be contracted as

$$
A^{\mathrm{R}}=\frac{16 q_{0}}{\pi^{6} D} \frac{1+\frac{17 \pi^{2} h^{2}}{84(1-v) a b} f(a, b, x, y)}{1+\frac{\pi^{2} h^{2}}{420(1-v) a b} f(a, b, x, y)} .
$$

The terms $a / b$ in Eq. (10) and $h / \sqrt{a b}$ in Eq. (11) indicate the plate aspect ratio and its relative thickness, respectively. For a square plate, these reduce to $a / b=1$ and $h / a$. Using Eq. (11) and equating the Mindlin and Reddy plate deflection gives

$$
\begin{aligned}
1 & +\frac{\pi^{2} h^{2}}{420(1-v) a b} f(a, b, x, y)+\frac{\pi^{2} h^{2}}{6 \kappa(1-v) a b} f(a, b, x, y) \\
& +\frac{\pi^{4} h^{4}}{2520 \kappa(1-v)^{2} a^{2} b^{2}}(f(a, b, x, y))^{2} \\
& =1+\frac{17 \pi^{2} h^{2}}{84(1-v) a b} f(a, b, x, y) .
\end{aligned}
$$

Hence, the usual shear correction factor of

$$
\kappa=\frac{5}{6}
$$

is obtained from Eq. (12) if the highest order term is neglected. Taking into account the highest order term, we have the exact shear correction factor

$$
\kappa=\frac{5}{6}\left[1+\frac{\pi^{2}}{420(1-v)} \frac{h^{2}}{a b} f(a, b, x, y)\right] .
$$

Since the maximum deflection takes place at the plate center, it is practical to consider the shear correction factor there, i.e.,

$$
\kappa=\frac{5}{6}\left[1+\frac{\pi^{2}}{420(1-v)} \frac{h^{2}}{a b} f\left(a, b, \frac{a}{2}, \frac{b}{2}\right)\right]
$$

where the function described by Eq. (10) becomes

$$
f\left(a, b, \frac{a}{2}, \frac{b}{2}\right)=\frac{\sum_{m=1}^{\infty} \sum_{n=1}^{\infty} \frac{(-1)^{\frac{m+n}{2}}-1}{m n\left(\frac{b}{a} m^{2}+\frac{a}{b} n^{2}\right)}}{\sum_{m=1}^{\infty} \sum_{n=1}^{\infty} \frac{(-1)^{\frac{m+n}{2}}-1}{m n\left(\frac{b}{a} m^{2}+\frac{a}{b} n^{2}\right)^{2}}}
$$

at the plate center. For an extremely long and narrow plate, Eq. (15) reduces to

$$
\lim _{\frac{a}{b} \rightarrow \infty} f\left(a, b, \frac{a}{2}, \frac{b}{2}\right)=\frac{a}{b}
$$

We note that both the numerator and denominator of Eq. (15) are dependent on the plate aspect ratio $a / b$. Setting $a \geq b$ for the uniformly loaded plate, Table 1 lists the denominator and numerator of Eq. (15) by performing double series summation. The summation was performed up to $m=n=41$ in order to obtain sufficient numerical accuracy.

A simple curve fit based on Table 1 gives the function

$$
\begin{aligned}
f\left(a, b, \frac{a}{2}, \frac{b}{2}\right)= & -0.0062\left(\frac{a}{b}\right)^{3}+0.1281\left(\frac{a}{b}\right)^{2} \\
& +0.1956\left(\frac{a}{b}\right) \\
& +1.4598 ; \quad ; \leq a / b \leq 10
\end{aligned}
$$

with a statistical accuracy of $R^{2}=0.9998$. 
Table 1 Computed results of Eq. (15)

\begin{tabular}{lccc}
\hline Plate aspect ratio $\frac{a}{b}$ & Numerator $\sum_{m=1}^{\infty} \sum_{n=1}^{\infty} \frac{(-1)^{\frac{m+n}{2}}-1}{m n\left(\frac{b}{a} m^{2}+\frac{a}{b} n^{2}\right)}$ & Denominator $\sum_{m=1}^{\infty} \sum_{n=1}^{\infty} \frac{(-1)^{\frac{m+n}{2}-1}}{m n\left(\frac{b}{a} m^{2}+\frac{a}{b} n^{2}\right)^{2}}$ & Ratio $f\left(a, b, \frac{a}{2}, \frac{b}{2}\right)$ \\
\hline 1.0 & 0.44895 & 0.24409 & 1.839281 \\
1.2 & 0.44059 & 0.23578 & 1.868649 \\
1.5 & 0.40575 & 0.20627 & 1.967082 \\
2.0 & 0.34749 & 0.15215 & 2.283865 \\
3.0 & 0.24622 & 0.08167 & 3.014816 \\
5.0 & 0.15405 & 0.031175 & 4.941460 \\
7.5 & 0.10389 & 0.013907 & 7.470339 \\
10.0 & 0.07859 & 0.007824 & 10.04473 \\
\hline
\end{tabular}

\section{Sinusoidal load}

Suppose the load distribution takes a sinusoidal form

$$
q=q_{0} \sin \frac{m \pi x}{a} \sin \frac{n \pi y}{b}
$$

instead of being uniformly distributed-whereby $m$ and $n$ quantify the load waviness along the $x$ and $y$ axes, respectively-then Eqs.(10) and (14a) reduce to

$$
f(a, b, x, y)=\frac{b}{a} m^{2}+\frac{a}{b} n^{2}
$$

and

$$
\kappa=\frac{5}{6}\left[1+\frac{\pi^{2}}{420(1-v)} \frac{h^{2}}{a b}\left(\frac{b}{a} m^{2}+\frac{a}{b} n^{2}\right)\right]
$$

respectively. Although Eq. (20a) can be written in a simpler way as

$$
\kappa=\frac{5}{6}\left[1+\frac{\pi^{2} h^{2}}{420(1-v)}\left(\frac{m^{2}}{a^{2}}+\frac{n^{2}}{b^{2}}\right)\right],
$$

the former is instructive for showing the effect of relative plate thickness $h / \sqrt{a b}$ and aspect ratio $a / b$, in addition to Poisson's ratio. Unlike the previous section on uniform load, this section on sinusoidal load allows one to observe the interlacing effect of load waviness pattern and plate aspect ratio on the shear correction factor.

For the special case of square plate, perusal to Table 2 shows that load waviness increases the shear correction factor. Reference to the same table also shows that waviness is strongly influenced by the aspect ratio of the plate; the effect of load waviness along the longer side diminishes as the plate becomes long or very narrow, i.e.,

$$
\begin{array}{ll}
\kappa \approx \frac{5}{6}\left[1+\frac{n^{2} \pi^{2}}{420(1-v)} \frac{h^{2}}{b^{2}}\right] ; & a>>b \\
\kappa \approx \frac{5}{6}\left[1+\frac{m^{2} \pi^{2}}{420(1-v)} \frac{h^{2}}{a^{2}}\right] ; & a<<b
\end{array}
$$

and consequently, the relative thickness is governed by the ratio of the plate thickness to its shorter side.

\section{Results and discussion}

In determining the range of relative thickness that is applicable for the shear deformation theories, one may refer to Steele and Balch (2009) who classified the plate thickness into four categories: (i) $a / h>100$, (ii) $20<a / h$ $<100$, (iii) $3<a / h<20$, and (iv) $a / h<3$. This implies that one may then adopt the membrane theory for $h / a<0.01$, CPT for $h / a<0.05$, shear deformation theories for $h / a<$ 0.3333 , and elasticity theory for $h / a>0.3333$. It therefore follows that the TSDT-based shear correction factor for FSDT problems are therefore applicable for relative thickness range of $h / a<0.3333$. As such, the following results were computed for relative thickness up to 0.2 since shear deformation theories are not applicable for relative thickness of $1 / 3$ and above. As with the CPT and FSDT, the TSDT is applicable for auxetic materials since the development of these theories are not confined to cases where Poisson's ratio is positive.

\section{Uniform load}

Figure 2(a) and (b) shows the effect of relative plate thickness and plate aspect ratio on the shear correction

Table 2 Shear correction factor expressions for special cases of rectangular plates under sinusoidal loads

\begin{tabular}{lll}
\hline Square plates $a=b$ & Simple sinusoidal load distribution $(m=n=1)$ & General sinusoidal load distributions $(m, n \geq 1)$ \\
Rectangular plates $a \neq b$ & $k=\frac{5}{6}\left[1+\frac{\pi^{2}}{210(1-v)} \frac{h^{2}}{a^{2}}\right]$ & $k=\frac{5}{6}\left[1+\frac{\pi^{2}}{420(1-v)} \frac{h^{2}}{a^{2}}\left(m^{2}+n^{2}\right)\right]$ \\
& $k=\frac{5}{6}\left[1+\frac{\pi^{2}}{420(1-v)} \frac{h^{2}}{a b}\left(\frac{b}{a}+\frac{a}{b}\right)\right]$ & $k=\frac{5}{6}\left[1+\frac{\pi^{2}}{420(1-v)} \frac{h^{2}}{a b}\left(\frac{b}{a} m^{2}+\frac{a}{b} n^{2}\right)\right]$
\end{tabular}



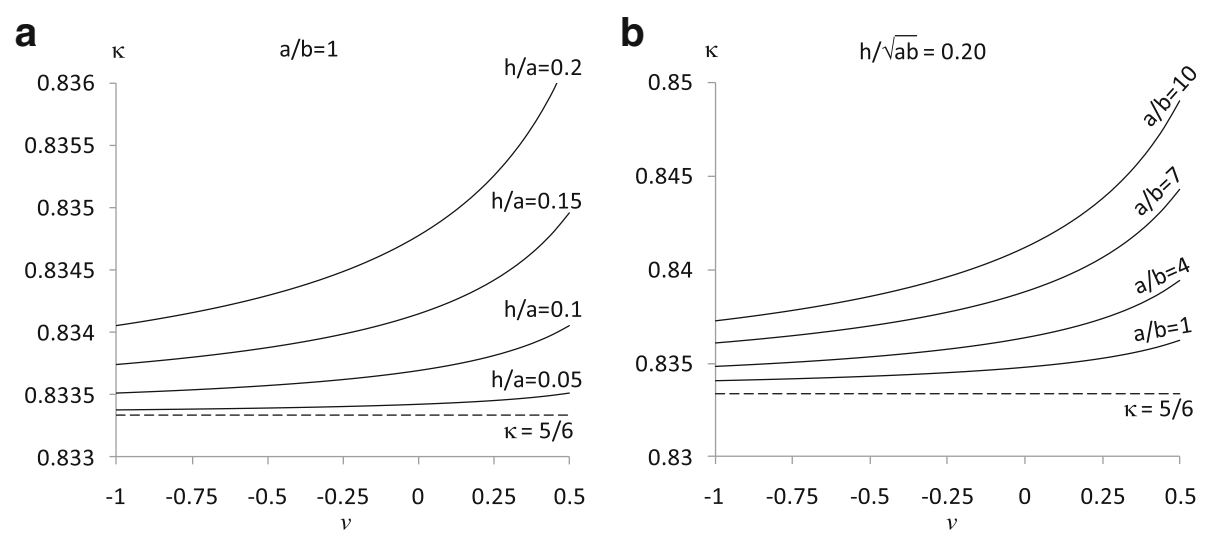

Fig. 2 Shear correction factor versus Poisson's ratio of a simply supported rectangular plate under uniform load with a variation in relative thickness for a square plate and $\mathbf{b}$ variation in aspect ratio for a thick plate

factor of a uniformly loaded plate for the entire range of Poisson's ratio. Specifically, the shear correction factor increases when (i) the plate becomes thicker, (ii) the plate becomes longer or narrower, and (iii) Poisson's ratio of the plate material is greater. The curves of the shear correction factors are plotted for Poisson's ratio of the range $-1 \leq v \leq 0.5$. This range is applicable for isotropic materials, in which solids of negative Poisson's ratio are termed "auxetic" materials (Lim, 2010, 2015a, 2016d); no bounds exist for Poisson's ratio of anisotropic ones (Ting, 2005; Lim, 2015b; Boldrin et al., 2016). The dashed lines in this and subsequent figures indicate the lower bound for the shear correction factor, i.e., $k=5 / 6$, for comparison. The influence of the plate geometry, in terms of the in-plane aspect ratio and the relative thickness, on the shear correction factor is plotted in Fig. 3 for $v=0.3$.

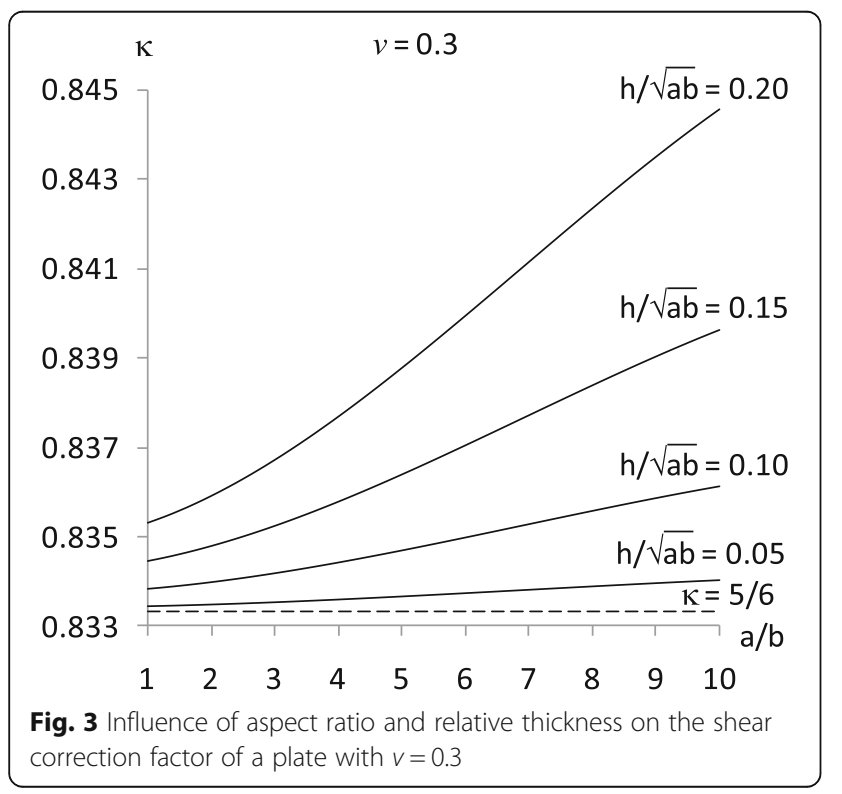

\section{Sinusoidal load}

In the case of sinusoidal load, there is a qualitatively comparable trend in the effect of plate geometry (aspect ratio and relative thickness) and Poisson's ratio on uniform load. In addition, the waviness of the transverse static load increases the shear correction factor, as evidenced in Fig. 4 for square plates.

In the special case of square plates, the shear correction factor is unchanged when the load waviness changes direction. For example, the shear correction factor for $(m, n)=(3,1)$ is similar to that for $(m, n)=(1,3)$; likewise, the shear correction factor for $(m, n)=(5,1)$ is similar to that for $(m, n)=(1,5)$. This observation, however, does not hold for rectangular plates. Perusal to Eqs.(20) or (21) shows that for very long or very narrow plates, the load waviness measured along the shorter side has greater influence than that along the longer side, as shown in Fig. 5.

\section{Comparison with other cases}

This section makes two types of comparisons, i.e., (a) with plates of other shapes but with similar boundary condition and (b) with similar plates but other boundary conditions. To put into perspective the current results with other plates under similar boundary conditions, a comparison is made with some recently improved shear correction factors of very thick plates. Table 3 summarizes the improved shear correction factors of very thick plates evaluated at the plate centroid for three different Poisson's ratio within isotropic solids: extremely auxetic $(v=-1)$, typical solids $(v=0.3)$, and incompressible solids $(v=0.5)$. The plates considered for comparison are simply supported isosceles right triangular plate (Lim, 2016a), equilateral triangular plate (Lim, 2016b), square plate, and rectangular plate of aspect ratio 4 under uniform load and possess the dimensionless plate thickness of $h / a=0.2$. 

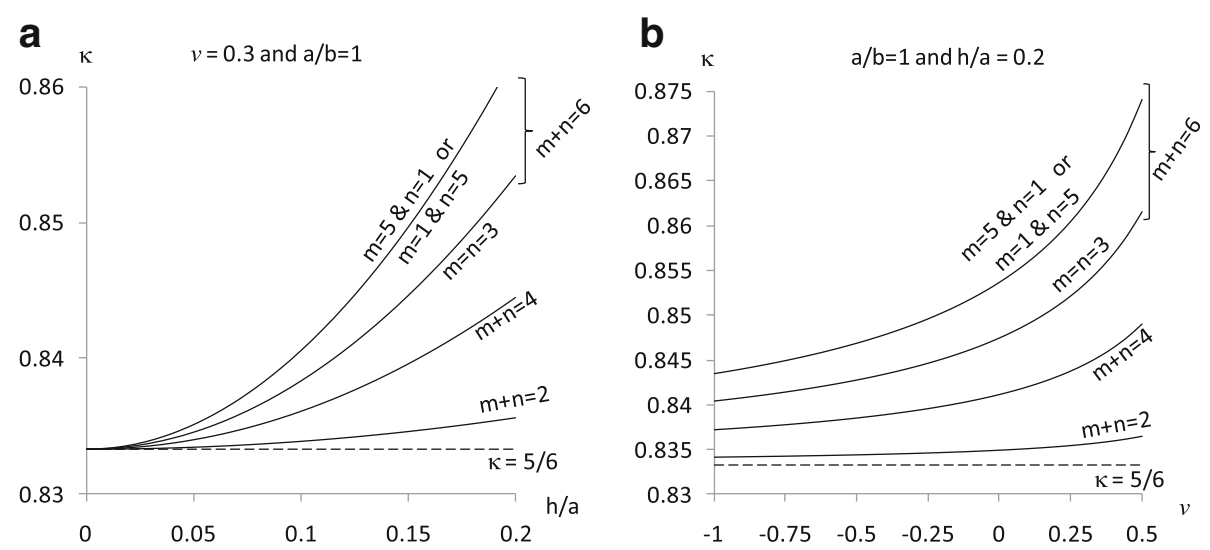

Fig. 4 Effect of load waviness on the shear correction factor of a sinusoidally loaded square plate with varying a relative thickness and b Poisson's ratio

The descriptions of shear correction factors developed herein apply only for rectangular plates of simply supported boundary condition, and are therefore not applicable for thick rectangular plates of clamped and/or free edges. Nevertheless, it is worthy to note that the FSDT for Levy plates, as reviewed by Wang et al. (2000), adopts a shear correction factor of $5 / 6$. Therefore, on this basis and on the basis of the results obtained from this paper, it can be said that the shear correction factor of $5 / 6$ could well continue to be a tight lower bound and that Poisson's ratio, alongside the plate's relative thickness, exerts influence on the shear correction factor.

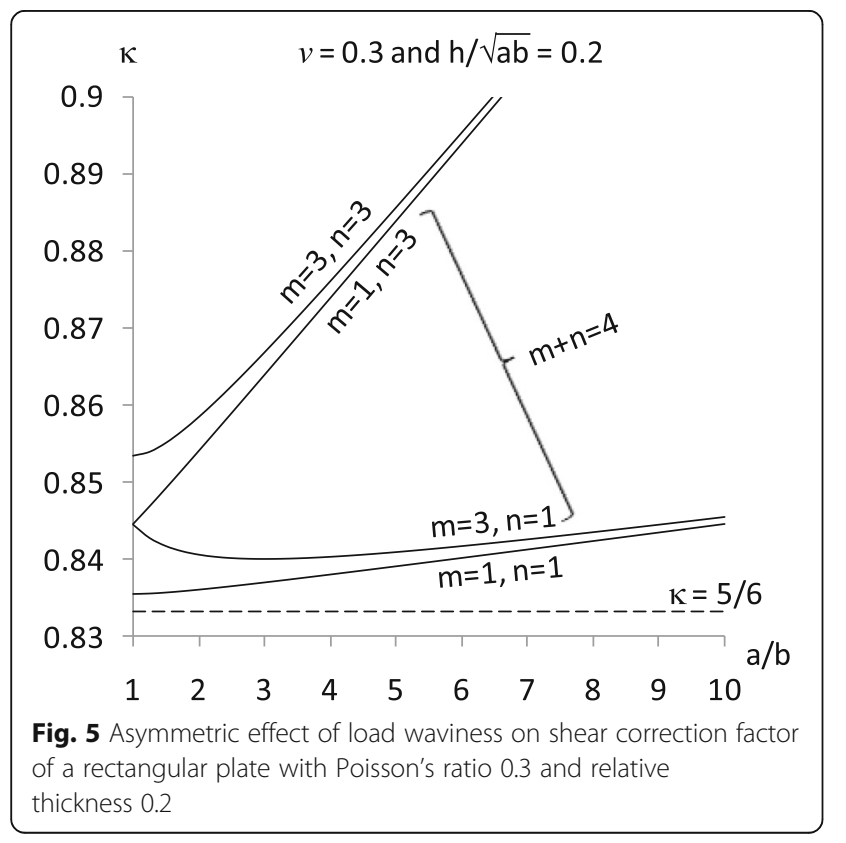

\section{Conclusions}

Exact shear correction factors for simply supported very thick rectangular plates under static loads have been developed herein for the case of uniform and sinusoidal loads using the Mindlin-Kirchhoff relationship and the Reddy-Kirchhoff relationship. Results obtained herein for uniform and sinusoidal loads show that the exact shear correction factor is higher than the commonly used shear correction factor of $5 / 6$ under the following conditions:

(a) Very thick plates

(b)Very long or narrow plates

(c) Plates made from large Poisson's ratio (especially incompressible materials)

(d)Highly patterned loading pattern or sinusoidal load with high load waviness

However, the use of the lower bound shear correction factor of $\kappa=5 / 6$ is valid under the following conditions:

(a) Marginally thick plates

(b)Square or almost square plates

(c) Plates made from auxetic materials, and

(d)Less wavy load pattern, especially uniform loads.

\section{Nomenclature}

$A=$ Amplitude of plate deflection

$a, b=$ In-plane dimensions of rectangular plate along $x, y$ axes

$D=$ Plate flexural rigidity

$E=$ Young's modulus

$G=$ Shear modulus

$h=$ Plate thickness

$M=$ Marcus moment

$m, n=$ Load waviness along $x, y$ axes

$q=$ Load intensity 
Table 3 Summary of shear correction factors of simply supported and uniformly loaded square and triangular plates with $h / a=0.2$

\begin{tabular}{lllll}
\hline Schematics of plates & $\begin{array}{l}\text { Extremely auxetic material } \\
(v=-1)\end{array}$ & $\begin{array}{l}\text { Typical conventional } \\
\text { material }(v=0.3)\end{array}$ & $\begin{array}{l}\text { Incompressible material } \\
(\mathrm{v}=1 / 2)\end{array}$ & Remarks \\
\hline$k=0.8351$ & $\kappa=0.8384$ & $k=0.8405$ & Isosceles right triangular plate (Lim \\
$2016 a)$
\end{tabular}

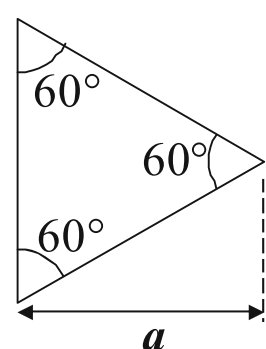

$K=0.8344 \quad K=0.8364 \quad K=0.8376 \quad$ Equilateral triangular plate (Lim 2016b)

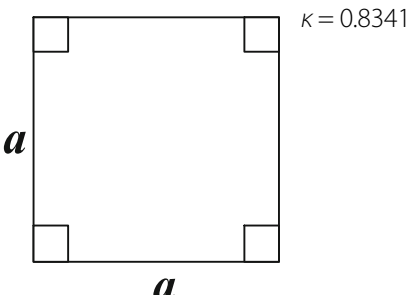

$K=0.8354 \quad K=0.8362 \quad$ Square plate (this paper)

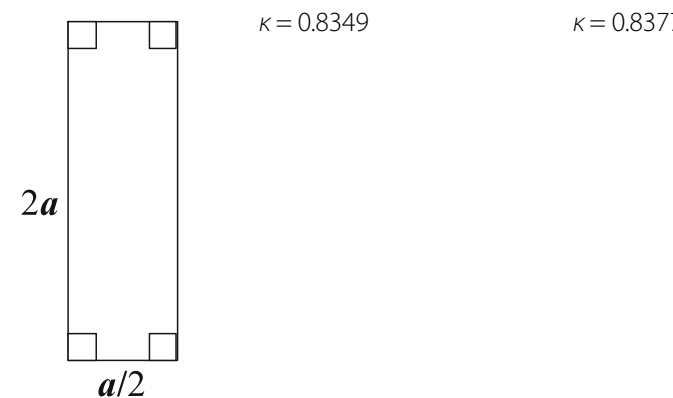

$K=0.8394 \quad$ Rectangular plate with aspect ratio of 4 (this paper)

$R^{2}=$ Coefficient of determination

$w=$ Plate deflection

$x, y=$ In-plane Cartesian coordinates

$\kappa=$ Shear correction factor

$v=$ Poisson's ratio

\section{Superscripts}

$K=$ Kirchhoff plate

$M=$ Mindlin plate

$R=$ Reddy plate

\section{Subscript}

$0=$ Maximum intensity for load.

\section{Competing interests}

The author declares that he has no competing interests.

Received: 22 June 2016 Accepted: 3 November 2016

Published online: 25 November 2016

\section{References}

Boldrin, L., Hummel, S., Scarpa, F., Di Maio, D., Lira, C., Ruzzene, M., Remillat, C. D.

L., Lim, T. C., Rajasekaran, R., \& Patsias, S. (2016). Dynamic behaviour of auxetic 
gradient composite hexagonal honeycombs. Composites Structures, $149,114-124$

Chan, K. T., Lai, K. F., Stephen, N. G., \& Young, K. (2011). A new method to determine the shear coefficient of Timoshenko beam theory. Journal of Sound and Vibration, 330(14), 3488-3497.

Dong, S. B., Alpdogan, C., \& Taciroglu, E. (2010). Much ado about shear correction factors in Timoshenko beam theory. International Journal of Mechanical Sciences, 47(13), 1651-1655.

Han, S. M., Benaroya, H., \& Wei, T. (1999). Dynamics of transversely vibrating beams using four engineering theories. Journal of Sound and Vibration, 225(5), 935-988.

Hlavacek, I., \& Chleboun, J. (2000). Reliable analysis of transverse vibrations of Timoshenko-Mindlin beams with respect to uncertain shear correction factor. Computer Methods in Applied Mechanics and Engineering, 190(8-10), 903-918.

Hull, A. J. (2005). An exact analytical expression of the shear coefficient in the Mindlin plate equation. Journal of the Acoustical Society of America, 117(4), 2601-2601.

Hull, A. J. (2006). Mindlin shear coefficient determination using model comparison. Journal of Sound and Vibration, 294(1\&2), 125-130.

Hutchinson, J. R. (1980). Vibrations of solid cylinders. ASME Journal of Applied Mechanics, 47(4), 901-907.

Hutchinson, J. R. (2001). Shear coefficients for Timoshenko beam theory. ASME Journal of Applied Mechanics, 68(1), 87-92.

Lee, K. H., Lim, G. T., \& Wang, C. M. (2002). Thick Levy plates re-visited. International Journal of Solids and Structures, 39(1), 127-144.

Lim, T. C. (2010). In-plane stiffness of semiauxetic laminates. ASCE Journal of Engineering Mechanics, 136(9), 1176-1180.

Lim, T. C. (2015a). Shear deformation in beams with negative Poisson's ratio. MechE Journal of Materials Design and Applications, 229(6), 447-454.

Lim, T. C. (2015b). Auxetic Materials and Structures. Singapore: Springer.

Lim, T. C. (2016a). Refined shear correction factor for very thick simply supported and uniformly loaded isosceles right triangular auxetic plates. Smart Materials and Structures, 25(5), 054001

Lim, T. C. (2016b). Higher order shear deformation of very thick simply-supported equilateral triangular plates under uniform load. Mechanics Based Design of Structures and Machines, 44(4), 514-522.

Lim, T. C. (2016c). Combined effect of load waviness and auxeticity on the shear deformation in a class of rectangular plates. IOP Conference Series: Materials Science and Engineering, 157, 012011.

Lim, T. C. (2016d). Large deflection of circular auxetic membranes under uniform load. ASME Journal of Engineering Materials and Technology, 138(4), 041011.

Pai, P. F., Anderson, T. J., \& Wheater, E. A. (2000). Large-deformation tests and total-Lagrangian finite-element analyses of flexible beams. International Journal of Solids and Structures, 37(21), 2951-2980.

Pai, P. F., \& Schultz, M. J. (1999). Shear correction factors and an energy-consistent beam theory. International Journal of Solids and Structures, 36(10), 1523-1540.

Popescu, B., \& Hodges, D. H. (2000). On asymptotically correct Timoshenko-like anisotropic beam theory. International Journal of Solids and Structures, 37(3), $535-558$

Puchegger, S., Bauer, S., Loidl, D., Kromp, K., \& Peterlik, H. (2003). Experimental validation of the shear correction factor. Journal of Sound and Vibration 261(1), 177-184.

Rössle, A. (1999). On the derivation of an asymptotically correct shear correction factor for the Reissner-Mindlin plate mode. Comptes Rendus de l'Académie des Sciences - Series I - Mathematics, 328(3), 269-274.

C. R. Steele, C. D. Balch (2009) Introduction to the Theory of Plates. Stamford University. http://web.stanford.edu/ chasst/Course\%20Notes/ Introduction\%20to\%20the\%20Theory\%20of\%20Plates.pdf. Accessed 24 October 2016.

Stephen, N. G. (1997). Mindlin plate theory: best shear coefficient and higher spectra validity. Journal of Sound and Vibration, 202(4), 539-553.

Ting, T. C. T. (2005). Poisson's ratio for anisotropic elastic materials can have no bounds. Quarterly Journal of Mechanics and Applied Mathematics, 58(1), 73-82.

Wang, C. M., Reddy, J. N., \& Lee, K. H. (2000). Shear Deformable Beams and Plates. Oxford: Elsevier.

Yu, W., \& Hodges, D. H. (2004). Elasticity solutions versus asymptotic sectional analysis of homogeneous, isotropic, prismatic beams. ASME Journal of Applied Mechanics, 71(1), 15-23.

\section{Submit your manuscript to a SpringerOpen ${ }^{\circ}$ journal and benefit from:}

- Convenient online submission

- Rigorous peer review

- Immediate publication on acceptance

- Open access: articles freely available online

- High visibility within the field

- Retaining the copyright to your article

Submit your next manuscript at $\gg$ springeropen.com 